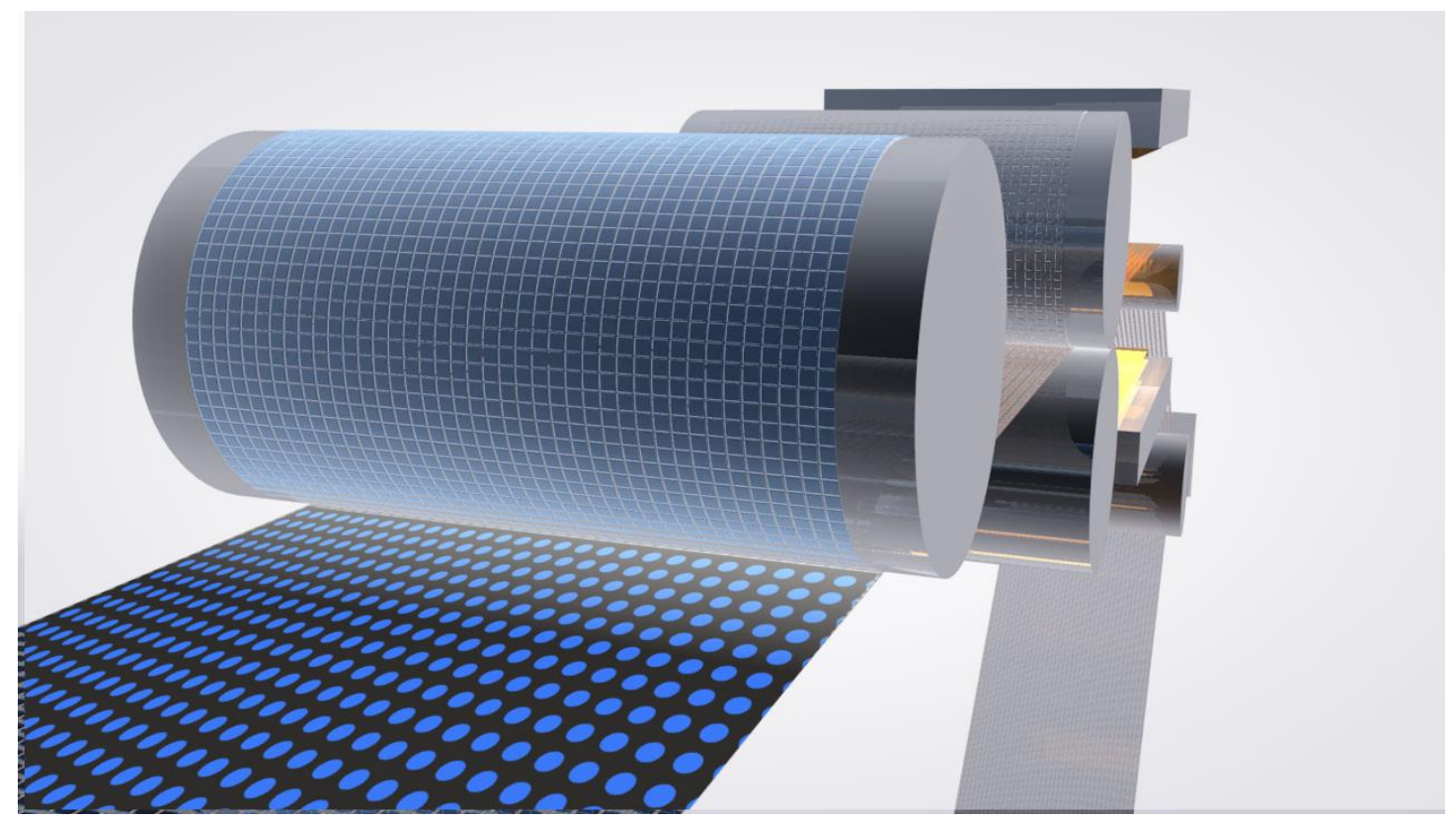

Nanofabrication Technologies for Roll-to-Roll Processing

Report from the NIST-NNN Workshop

September 27-28, 2011

Edited by Jeffrey D. Morse, Ph.D. 


\title{
Nanofabrication Technologies for Roll-to-Roll Processing
}

\author{
Report from the NIST-NNN Workshop September 27-28, 2011
}

Edited by:

Dr. Jeffrey D. Morse, Managing Director, National Nanomanufacturing Network

Workshop organizing committee:

Dr. Christopher Soles, NIST

Prof. James Watkins, University of Massachusetts Amherst

Dr. Jeffrey Morse, NNN

Dr. Thomas Wiggins, NIST

Dr. Michael Schen, NIST

Workshop participants;

Ken Carter

Paul Clark

Rick Daniels

Xian Du

Jennifer Ernst

Gregg M. Gallatin

Dan Gamota

Amit Goyal

Jay Guo

David Hardt

Michael Hunter

James Kadtke

Nikolaos Kehagias

Hong-Yee Low

Trevor Niblock

Joe Petrzelka

Mark Poliks

Robert Praino

George Ranuik

Dennis Slafer

Chris Soles

S.V. Sreenivasan

Ganesh Sundaram

James Watkins

Lloyd Whitman

Michael Wright
University of Massachusetts Amherst

University of Massachusetts Amherst

Carestream Advanced Materials

Massachusetts Institute of Technology

Thin Film Electronics ASA

National Institute of Standards and Technology

Printovate Technologies

Oak Ridge National Laboratory

University of Michigan

Massachusetts Institute of Technology

Liquidia Technologies

National Nanotechnology Coordination Office

Catalan Institute of Nanotechnology

Institute of Materials Research and Engineering

Magzor Corporation

Massachusetts Institute of Technology

State University of New York Binghamton

Chasm Technologies

Carpe Diem Technologies

MicroContinuum, Inc.

National Institute of Standards and Technology

University of Texas Austin/Molecular Imprints, Inc.

Cambridge NanoTech, Inc.

University of Massachusetts Amherst

National Institute of Standards and Technology

University of Massachusetts Amherst 


\section{Table of Contents}

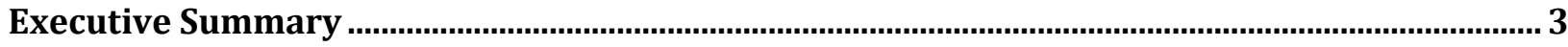

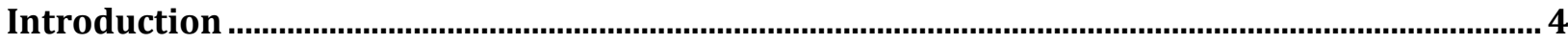

Potential Benefits of Nanofabrication Processes for Roll-to-Roll................................................................... 4

Workshop on Nanofabrication Technologies for Roll-to-Roll Processing........................................................... 5

Pre-Workshop Questionnaire ...........................................................................................................................

Workshop Organization and Format .................................................................................................................... 6

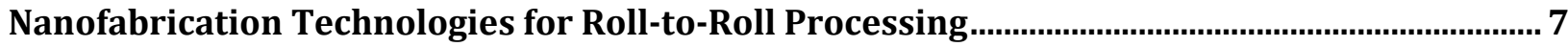

Industry Assessment: International Electronics Manufacturing Initiative (iNEMI) ...................................

Functional Inks for Active and Passive Devices ........................................................................................ 8

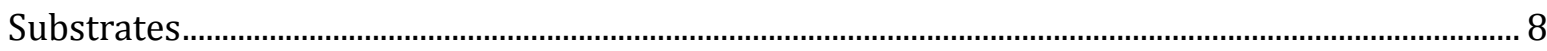

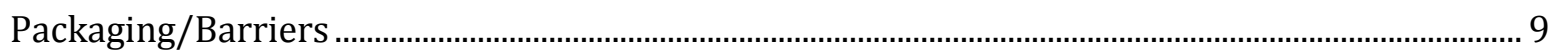

Manufacturing Platforms and Processing Equipment ............................................................................ 9

Testing and Quality Control Tools ........................................................................................................ 9

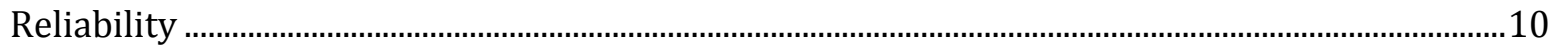

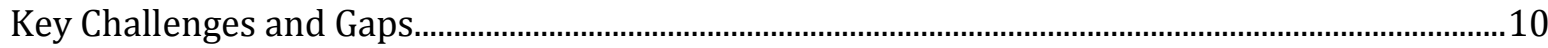

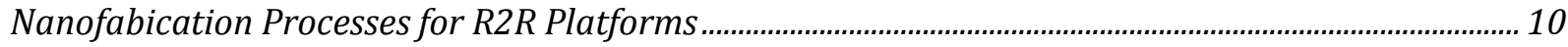

Solution Casting of Functional Inks ....................................................................................................10

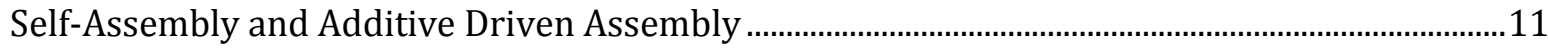

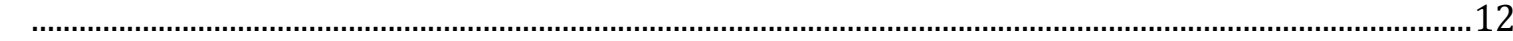

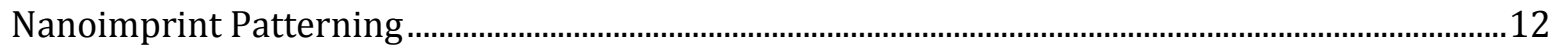

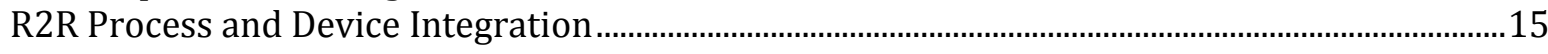

Emerging Tools, Capability, and Infrastructure for R2R Platforms..............................................17

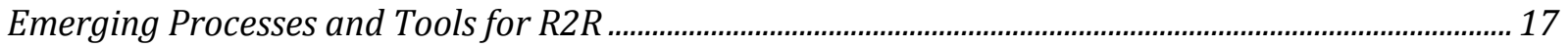

Atomic Layer Deposition.................................................................................................................17

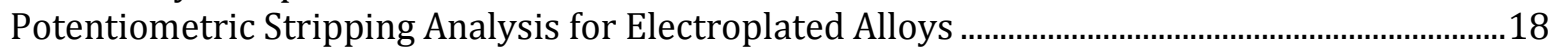

High Temperature Roll-to-Roll Processes .................................................................................................18

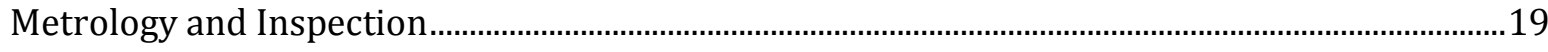

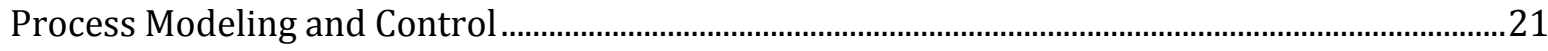

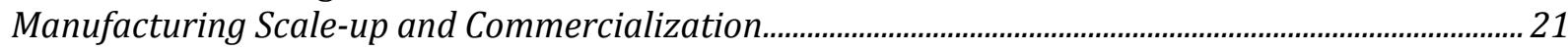

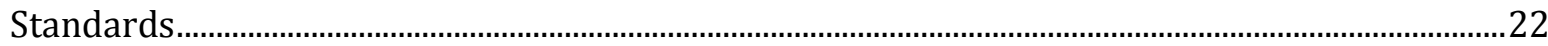

Accelerating Technology Transfer and Advanced Manufacturing Scale-up.......................................22

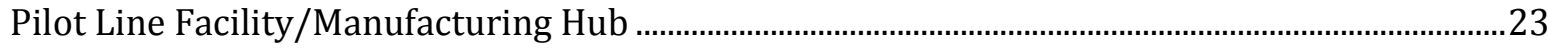

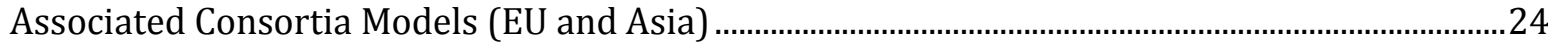

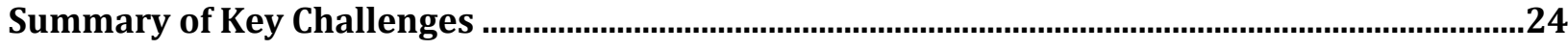

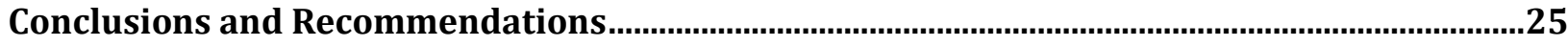

Appendix A: Participant Response to Workshop Questionnaire ..................................................26

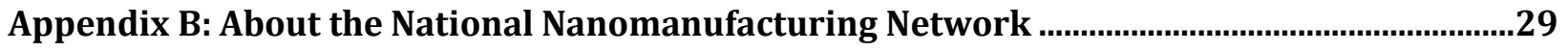

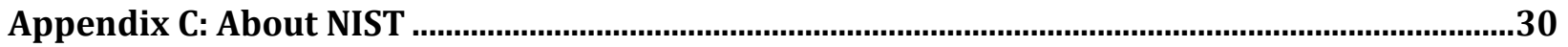




\section{Executive Summary}

The roll-to-roll (R2R) platform is an industrial vetted way to handle solution-based processes and coatings for high volume manufacturing. R2R processes are implemented for applications as diverse as instant photographic film, separation membranes, filtration media, advance printing and holographic coatings, polymer anti-shatter films for car windshields, flexible solar panels, composite electrodes on metal foils for lithium-ion batteries, and macroscale patterning of metal interconnects for flexible packaging of electronic components. Currently, manufacturers are looking for new innovative continuous-feed processes for printing materials and structures onto roll-based flexible substrates. In particular there is considerable interest in adapting $\mathrm{R} 2 \mathrm{R}$ technologies for the extreme miniaturization of critical feature sizes to the nanoscale. This intersection of nanofabrication with $\mathrm{R} 2 \mathrm{R}$ processes has considerable potential to spur innovation and economic growth. Nanofabrication for R2R process platforms represents a disruptive manufacturing technology involving solution-processed, multi-layer precision coatings with functionalized nanostructures, materials, and patterning capability to realize unprecedented properties and functionalities for next generation consumer products. Commercial products and applications impacted by this approach include displays, lighting, energy storage, electronics, solar photovoltaics. These high-value consumer electronics predicted to see double-digit market growth beyond the next decade (IDTechEx 2009-2029 Market Report). Incorporation of emerging nanofabrication methods within R2R manufacturing processes will make it possible to economically generate high value-added technology products at meters-per-minute rates on plastic film, paper, or foil, achieving feature dimensions as small as ten nanometers over areas encompassing billions of identical devices. Meeting this challenge is a key to high-rate manufacturing of nano-enabled products and for establishing viable industrial-scale manufacturing platforms for continuous large-area roll-to-roll processing.

This workshop consisted of a group of 30 experts and stakeholders in the field of R2R processing convened by NIST and the National Nanomanufacturing Network (NNN) to discuss the state of nanofabrication in $\mathrm{R} 2 \mathrm{R}$ processes, as well as to identify significant barriers to implementation of emerging process methods and materials. The workshop took place on September 27-28, 2011 at the Seaport Convention Center in Boston in conjunction with the Nanomanufacturing Summit 2011. The agenda included welcoming remarks followed by plenary talks on the nanotechnology roadmap for the International Electronics Manufacturing Initiative ( $i \mathrm{NEMI})$, and translational bottom-up nanomanufacturing methods for $\mathrm{R} 2 \mathrm{R}$, with the remainder of the agenda including talks covering a range of nanofabrication methods and topics relevant to $\mathrm{R} 2 \mathrm{R}$ process platforms. The participants, representing tool vendors, contract manufacturers, materials suppliers, government, and academia, discussed present status and ongoing development of nanofabrication processes being scaled to R2R platforms. The group also addressed barriers to the commercialization of nanomaterials and nanmanufacturing approaches.

The workshop participants successfully identified key barriers to the adoption of nanofabrication processes within R2R manufacturing platforms, as well as to accelerating the commercialization of nanoenabled flexible electronics consumer products. Several approaches were identified that would enhance U.S. competitiveness in this area, including the need for standards to enable product integration across emerging supply lines, improved metrology and in-line characterization, scaled process control and modeling, new materials, and improved off-line characterization tools for product development. Discussants considered the establishment of infrastructure, including possible shared demonstration facilities, where academic research could be transferred and scaled to industrially meaningful process scales, working closely with industry to accelerate the adoption of advanced nanomanufacturing processes. The goal of such a consortium or industry hub would be to enhance broad based industry growth impacting numerous product and application areas, as well as advance the performance of key intermediate technologies (e.g; barrier films, transparent conductors, high- $k$ dielectrics, etc.) that impact different product applications. Creation of an industry network to further elucidate and resolve such critical issues would be advantageous to U.S. competitiveness in this area. 


\section{Introduction}

Nanofabrication methods are essential elements in the field of nanomanufacturing, which remains the essential bridge between the discoveries of the nanosciences and real-world nanotechnology products. Advancing nanotechnology from the laboratory into high-volume production ultimately requires careful study of manufacturing system issues including product design, reliability and quality, process design and control, shop floor operations and supply chain management. Nanomanufacturing is the controllable manipulation of materials structures, components, devices, and systems at the nanoscale (1 to 100 nanometers) in one, two, and three dimensions for large-scale reproducibility of value-added components and devices. Nanofabrication methodologies encompass bottom-up directed assembly, top-down highresolution processing, molecular systems engineering, and hierarchical integration with larger scale systems. As dimensional scales of materials and molecular systems approach the nanoscale, the conventional rules governing the behavior and properties of these components, devices, and systems change significantly. As such, the behavior of the final product is enabled by the collective performance of the nanoscale building blocks.

Roll-to-roll (R2R) processing is a broadly defined terminology encompassing a range of processes wherein a substrate is transferred between two moving rolls during which the processes are applied to the substrate. As such, the processes have both a time and spatial occurrence, requiring specific customization to achieve the desired process outcomes. Inherently, $\mathrm{R} 2 \mathrm{R}$ processes require flexible substrates, which generally entail plastic or polymer materials, and in limited cases, can also include thin-form glass, metal, or ceramic substrates. Manufacturing processes possible in R2R platforms include the full range of additive and subtractive processes, including vacuum-, thermal-, and solution-based methods. Notwithstanding the range and types of processes possible on $\mathrm{R} 2 \mathrm{R}$ platforms, the inherent advantage for many product applications requiring high-rate and low-cost is the economy of scale offered by solution-based processes. R2R manufacturing has transitioned from paper and textiles to advanced multi-layer coatings as exemplified by products from companies such as Polaroid and Kodak for imaging and film products and applications. More recently, new industries with applications in printed electronics have emerged. Initially, these have been in the area of semiconductor integrated circuit packaging and printed circuit board replacement, and are now being considered for a range of integrated device and system applications requiring higher levels of functionality and performance printed directly on the flexible substrate. The printed electronics industry has expanded into a broad range of market applications including flexible electronics, displays, touch screens, sensors, and energy harvesting and storage.

\section{Potential Benefits of Nanofabrication Processes for Roll-to-Roll}

Nanofabrication processes represent an advanced manufacturing technology having the potential to impact a broad range of flexible electronics markets and applications. Nanofabrication technologies were initially developed as batch-type processes for the semiconductor industry, but they are not limited to batch processing. By coupling nanofabrication techniques to existing infrastructures and production platforms such as $\mathrm{R} 2 \mathrm{R}$, it is possible to imagine a new manufacturing paradigm with the potential to to enhance U.S. competitiveness. Considered a game changer by industry for progress in printed electronics, this intersection of two technologies - volume production on continuous rolls of film, and nanofabrication technologyopens up many industry market opportunities. The diverse product applications of R2R range high on the value chain. They include not only electronic and energy devices such as solar cells, 
sensors, antennas, memory, displays, capacitors and batteries, but also many other functional materials such as barrier layers, security films, transparent conductor layers, magnetic metamaterials, chem/bio shielding, water-repellent surfaces, filtration/separation membranes and other products incorporating nanomaterials. Figure 1 shows market projections for printed electronics in a range of commercial product areas. As a disruptive manufacturing technology, R2R nanomanufacturing has the opportunity to accelerate the growth rate, substantially increasing the value of nano-enabled components making up such market projections. Roll-toroll nanomanufacturing comes with many benefits. These include low unit costs, high-volume rates of production, process efficiency through sequential application of successive layers on a web, energy and materials efficiency, versatility in the types of devices that can be made, formfactor benefits of flexibility and low weight, and relatively benign and plentiful constituent chemicals and materials.

The PE market in 2010 is about $\$ 2 B$, about $20 \%$ of that is flexible. By 2020 , that rises to almost $\$ 60 \mathrm{~B}, 75 \%$ of that is flexible.

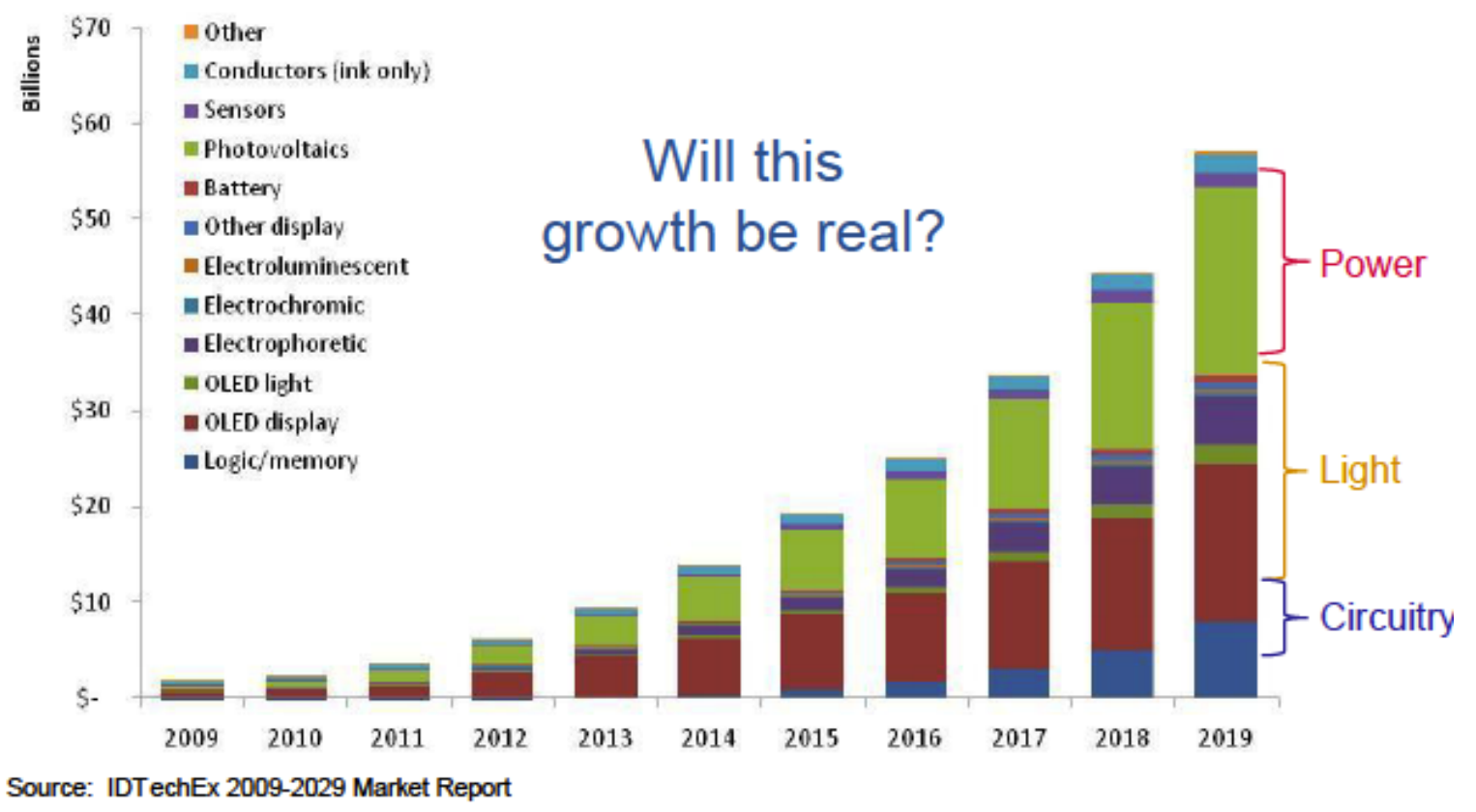

Figure 1: Global market projections for flexible electronics

\section{Workshop on Nanofabrication Technologies for Roll-to-Roll Processing}

The Workshop on NanofabricationTechnologies for Roll-to-Roll Processing, an Academic-Industry Workshop on Technologies for American Manufacturing Competitiveness co-sponsored by NIST and the NNN, invited researchers, institutions, and leading companies involved in roll-to-roll processing and scalable nanomanufacturing methodologies to identify and discuss challenges for successful merging of these technologies in advanced device manufacturing. The workshop included presentations by participants on relevant topics, along with discussion of general parameters for a multi-year technology roadmap to guide progress in roll-to-roll processing of nanoscale devices. 


\section{Pre-Workshop Questionnaire}

A pre-workshop questionnaire was provided in order to assist both participants in preparing presentations for the workshop, and organizers and participants in assessing the parameters for a technology roadmap covering roll-to-roll (R2R) nanomanufacturing. Questionnaire responses also aided in drafting this workshop report by bringing out pressing technical and infrastructural needs facing different facets of the technology sector, as well as federating portions of various related roadmaps that impact the broader industry landscape. This structured participant feedback may further be useful for both the NNN and NIST in terms of scoping and suggesting research agendas in various government and academic laboratory programs as well as future federal and state funding opportunities.

Five pre-workshop questions were;

1. What are the biggest barriers or process bottlenecks that need to be addressed to realize roll-to-roll nanomanufacturing?

2. Can you foresee enabling/emerging technologies that need to be developed in order to realize roll-to-roll nanomanufacturing?

3. Are there specific materials and/or metrology needs that are needed to realize rollto-roll nanomanufacturing?

4. What infrastructure needs to be developed to transition roll-to-roll technologies to volume manufacturing?

5. What sorts of partnerships (public, private, academic, consortia) are needed to accelerate progress towards volume manufacturing?

\section{Workshop Organization and Format}

The workshop brought together approximately thirty leading experts to identify key issues for nanofabrication in $\mathrm{R} 2 \mathrm{R}$ processes, and included an opening evening session with introductory remarks, two plenary presentations and overviews of $\mathrm{R} 2 \mathrm{R}$ nanomanufacturing approaches, followed by a full day of presentations and associated topical discussions. Participants prepared presentations and remarks addressing technical topics including:

- Generating low-cost, commodity-scale materials sets

- Demonstrating precision cooperative assembly

- Utilization of surface-directed/guided assembly of critical features via imprint stamping

- Fabrication of ordered hybrid nanocomposites at high rates

- Nanoimprint processes

- Process integration

- Online metrology

- Global R\&D efforts and developing manufacturing capabilities, especially in Europe and Asia 
Workshop information and presentations can be found online at the website http://www.internano.org/r2rworkshop where a list of participants, agenda, and presentations can be found. The workshop was organized in conjunction with the Nanomanufacturing Summit 2011 (http://www.internano.org/nms2011/).

The workshop's goal was to identify and discuss progress and challenges for successful merging of nanofabrication technologies into advanced device manufacturing. The workshop included discussions of general parameters for a multi-year technology roadmap to guide progress in roll-to-roll processing of nanoscale devices. Specific objectives of the workshop were to:

- Identify needs, challenges, and priorities for research and development relative to nanofabrication for $\mathrm{R} 2 \mathrm{R}$ processes

- Identify and prioritize strategies for catalyzing the transition of emerging R2R nanofabrication methodologies to industrial platforms

- Discuss R2R nanofabrication projects and capabilities already underway that address needs within specific industry or application sectors

- Produce a report for dissemination to relevant stakeholders that can serve as guidance for future workshops and initiatives in advanced manufacturing.

\section{Nanofabrication Technologies for Roll-to-Roll Processing}

Nanofabrication processes incorporate a range of advanced processes, tools, and materials that effectively provide a path towards commercially scalable manufacturing platforms. Such nanomanufacturing capabilities rely on top-down and bottom-up nanoscale processes, including nanoimprint and sub-wavelength lithography, guided selfassembly and directed patterning methodologies, molecular engineering, and hierarchical integration across multiple length scales. Future industrialization of this technology naturally requires scaling system properties while retaining the collective properties of the nanoscopic elements over macroscale dimensions. Meeting this challenge is a key to high-rate manufacturing of nano-enabled products and for establishing viable industrialscale manufacturing platforms for continuous large-area roll-to-roll processing.

\section{Industry Assessment: International Electronics Manufacturing Initiative ( $i$ NEMI)}

Daniel Gamota of Printovate Technologies discussed the intersection of R2R processing and nanotechnology from an industry perspective, including perspectives from the iNEMI roadmap. The printing and microelectronics industries are at the intersection of a new industry resulting from the merging of printing, functional inks, and microelectronics technologies: large-area flexible electronics. The driver for large-area flexible electronics is an economic motivation to move away from semiconductor fabrication approaches and to establish manufacturing platforms based on printing equipment that will provide a means for scaling with costs that increase at a rate several orders of magnitude below the rates historically observed for silicon IC manufacturing. Several of the often-stated benefits for printing are:

- Printing provides greater manufacturing economies of scale, when considering deposition of solution-processable materials versus vacuum deposition and 
photolithography for fabrication.

- The costs associated with fabricating devices and assembling products designed with several IC chip sets are at least an order of magnitude higher than those that can be realized when using functional inks (organic, organic/inorganic hybrid) and printing-based technologies.

- Manufacturing of electronics using solution-processing equipment in large-area form factors versus being limited in size (e.g., $300 \mathrm{~mm}$ wafers) will enable several ubiquitous intelligent product platforms.

Large-area flexible electronics will grow into a multibillion-dollar industry over the next decade and will revolutionize our view of electronics and how we as a society interact with intelligent and responsive systems. The unique properties of flexible electronics such as compliant structures, ultra-thin profiles, light weight and potential low cost and high reliability could have enormous impact on consumer electronics, aviation and space electronics, life sciences, military applications and telecommunications. Large-area flexible electronics will enable a broad range of devices and applications not possible today. One of the critical enabling technologies for large area flexible electronics is R2R processing. Today $\mathrm{R} 2 \mathrm{R}$ is practiced in many mature industries (paper, film, printing, etc.). Due to its attributes R2R high-volume manufacturing has been proposed for electronics as a means to lower the cost of non-Si wafer-based large-area flexible electronics, such as displays.

iNEMI, in establishing the roadmap for large area flexible electronics, has identified several key technologies at the intersection of nanofabrication processes and R2R platforms.

\section{Functional Inks for Active and Passive Devices}

Present printing methods are limited by solubility and processability of the functional ink as nanoparticles very typically have dispersion issues resulting in agglomerations. Additionally, polymer conductors/semiconductors have solubility and stability issues. A range of nanoparticle-based inks is presently available for conductor applications, and while some require sintering post-process, nanoparticle inks have lower sintering temperature requirements. While there exist several organic conductors (e.g. PANI, PEDOT), at present they exhibit 2-3 orders of magnitude higher resistivity. Dielectric films still need work on high and ultra-low permittivity materials. Semiconducting inks still have a long way to go to improve transport properties, but are beginning to exhibit performance improvements. Small molecule, organic semiconducting polymers have solubility issues, and while additive functional groups improve solubility but reduce semiconducting properties somewhat, this approach is promising. Inorganic and hybrid inks are showing the most promise for semiconductors, including metal oxide and chalcogenate thin films, compositional nanowires and carbon nanotubes.

\section{Substrates}

Substrate classes include polymer film, metal, paper, textiles, glass, and ceramics. Issues surrounding dimensional stability of substrates--for example, surface roughness, registration, and multilayer films/coating--limit processes that can be used. Defects remain a large concern, with the impact dependent on critical feature and surface area for a given process 
application. Nominally, reduced defects will add to substrate cost. Large-area metrology to measure defects will be critical in the next 5-10 years. Substrate thermal stability ( $\left.T_{g}, C T E\right)$ is critical to process development and manufacturing scale-up. It is better to develop lower temperature processes than to develop higher-temperature and more thermally stable substrates, as the latter will be expensive.

Thermal and mechanical stability, resistance to moisture, gas and vapor transmission, and solvents are important factors to consider in substrate selection. In addition, surface smoothness, surface energy, optical transparency, commercial availability and costs must be considered. As with most materials, costs increase with substrate material property demands. Depending on the requirements of the application, a different flexible substrate will be required and for the more demanding applications the substrate will almost certainly be a multilayer composite structure.

As examples, PET and PEN having excellent dimensional stability up to $150^{\circ} \mathrm{C}$ are being used for a range of display backplane, RFID applications, data storage and automotive electronic wiring applications. Kapton and metal foils are finding uses in photovoltaics and electronics, and paper is already beginning to be used with printed electronics and batteries, having a demonstrated compatibility with functional inks, good thermal dimensional control and a well-developed supply chain. In addition, nonwoven textiles or engineered fabrics can be imprinted in a similar manner as paper and other substrates, and are finding an emerging range of applications.

\section{Packaging/Barriers}

Barrier films and packaging still use vacuum coatings for the best performance. Multilayer organic/inorganic coatings utilizing a solution-based or hybrid process approach are presently the best. Water vapor and oxygen detection limits are also an issue for characterizing barrier coatings.

\section{Manufacturing Platforms and Processing Equipment}

Many new processes are designed for production on conventional standard manufacturing equipment, as for example in thick film processing, but require customized steps for new products. In many cases use of an existing tool can reduce the number of process steps. Processes that are compatible with $\mathrm{R} 2 \mathrm{R}$ platforms include gravure, flexography, offset litho printing, screen, inkjet, and microcontact printing. Thin film processing can use either vacuum- or solution-based coatings, with the latter being more scalable and economic.

\section{Testing and Quality Control Tools}

\section{In-line Characterization}

Techniques include imaging and feedback control loops (PLCs), process materials metering, and electrical and optical measurements.

\section{Off-line Characterization}

Methods include electrical, mechanical, materials and interface structure, and imaging/pattern quality. 


\section{Reliability}

Process reliability is critical for scaling to large-area, high-throughput manufacturing lines. As such, process modeling, control, and real-time monitoring is still needed for many emerging product applications. Additional needs are simulation and design tools, and design for manufacturing approaches for flexible electronics devices and systems.

\section{Key Challenges and Gaps}

iNEMI has identified the "Top Four" Needs and Gaps as;

1. In-line inspection and testing equipment

2. Simulation and design tools

3. Robust R2R, roll-fed, and large format manufacturing platforms

4. Higher performance inks (semiconducting, OLED, PV-active, etc.)

\section{Nanofabication Processes for R2R Platforms}

\section{Solution Casting of Functional Inks}

Solution casting of composite inks is one of the earliest examples of nanofabrication processes being implemented on flexible substrates via R2R platforms. It provides an excellent tutorial on some of the challenges for bridging the nanotechnology gap in transitioning from lab to fab. Polaroid alumnus Robert Praino, now with Chasm Technologies, discussed the issues of developing carbon nanotube inks, then adapting these functional inks for applications such as transparent conductors and battery electrodes. Fellow Polaroid alumnus Richard Daniels, now with Carestream Advanced Materials described a R2R solution-based coating platform incorporating silver nanostructures for multilayer $\mathrm{x}$-ray film production. Using this as the basis for product development of functional nanostructure/ink compositions, the first step to successful validation is access to quality nanostructured materials using high-purity synthesis techniques, achieving homogenous dispersion in solution and avoiding agglomeration. Solution preparation methods may utilize filtration schemes that allow passage of high aspect ratio particles yet trap unwanted materials, and coaters use solution delivery systems that do not damage fragile particles or cause agglomeration. Coating methods must enable uniform deposition, resulting in the desired distribution of particles and stability of high surface area materials. While challenges are dependent on various factors of the processes and materials being used, general steps to success include understanding the materials being used, appropriate solution preparation and delivery, selection of coating/patterning method, incorporation of multi-layer strategies for reduced cost/increased yields, and the establishment of continuous manufacturing process conditions. 

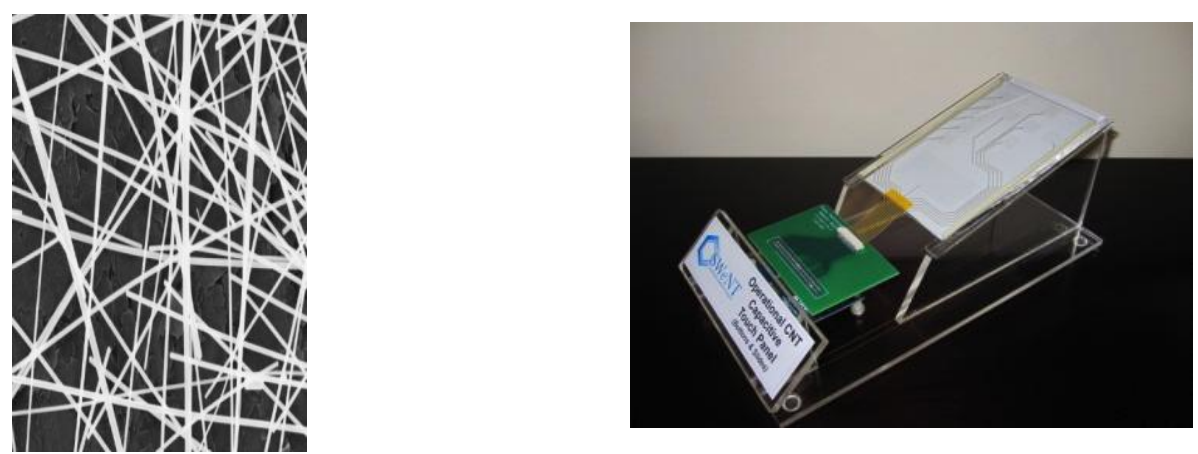

Figure 2: (left) Silver nanowire transparent electrodes. (right) An example of a carbon nanotube touch screen.

Key barriers in developing new materials for these applications include the need to first validate the processing and scalability of the materials, essentially determining that the process conditions do not change the material properties. Subsequent to process validation is generation of data for process controls. As existing manufacturing platforms are often hesitant to take the risk associated with new materials, these materials must establish a relevant platform capable of delivering an equivalent or better product (relative to the existing platform). As a result, materials companies typically face delayed access to the market, and bridging these process gaps is critical for sustainability in the case of small nanotechnology ventures. Additional barriers include identifying product specifications and requirements; understanding product performance tolerance to related defects; incorporating clean manufacturing protocols, rate control and purity levels for nano-morphologies; better understanding of and means for mitigating materials costs; ensuring that materials of interest are compatible with flexible supports and the required process methods; thermal and environmental stability of continuous, flexible substrates when processing functional layers; and incorporating barrier layers that minimize poisoning of functional layers or migration into flexible supports.

\section{Self-Assembly and Additive Driven Assembly}

Self-assembly is an established nanomanufacturing methodology whereby block copolymer (BCP) blends can be tailored to provide controlled domain size and ordered morphologies by appropriate selection of the molecular weight and fractional composition of the constituents in the blend. Upon casting on a surface having controlled properties, the polymers in the blend phase segregate during solvent annealing, forming cylindrical or lamellar domains having features ranging from 3-30 $\mathrm{nm}$. In this manner specific features or templates can be achieved through these process controls. An extension of this technique is referred to as directed self-assembly, and utilizes features either physically or chemically pre-patterned in the surface on which the BCP solution is cast. The pre-patterned features effectively anchor the BCP patterning during phase segregation, resulting in multiple ordered patterns for each pre-patterned feature. Using this technique, unprecedented pattern densities have been achieved, and this approach is now being utilized to create bit-patterned media for magnetic memory data storage.

An emerging nanomanufacturing technique referred to as additive-driven assembly enables block copolymer-nanoparticle (BCP-NP) solutions to be coated under certain conditions wherein the ordering and dispersion of the nanoparticles mimic that of the 
BCP morphology. Typically, such hybrid materials systems are formed through specific functionalization of the NPs, thereby facilitating bonding to targeted ligand sites within one of the polymer blocks. The NPs then follow the domain to which they are attached during phase segregation by solvent annealing of the BCP system, leading to highly ordered distribution and controlled density. Challenges in applying this method to practical applications include required modification to control both the NP loading in the polymer blend, as well as appropriate functionalization of the NPs to control the bonding sites for specific blocks of the BCP system without impacting the ability for the entire system to subsequently phase segregate and achieve order. Ultimately, the specific ligands to which the NPs bond will dictate their position within the ordered BCP domains, which may be critical for a given device or materials application.

James Watkins, director of the NSF Center for Hierarchical Manufacturing (CHM) at the University of Massachusetts Amherst, described the transition of these nanomanufacturing methods to R2R process platforms. Extension of self-assembly for high-volume fabrication of nanostructured materials and devices faces key issues with respect to materials availability and cost, as well as identification of and integration with high-impact technologies and products. Discussion of strategies to mitigate these issues included the availability of commodity-scale materials for low-cost/high-volume systems; the creation of technologically useful materials functionalized to realize electronic, mechanical, optical properties by design; the creation of well-ordered nanoparticle/BCP systems with prescriptive placement of NPs and high NP loadings; and the development of a robust $\mathrm{R} 2 \mathrm{R}$ manufacturing platform incorporating scalability, process models, manufacturability, metrology, QA, and process control.
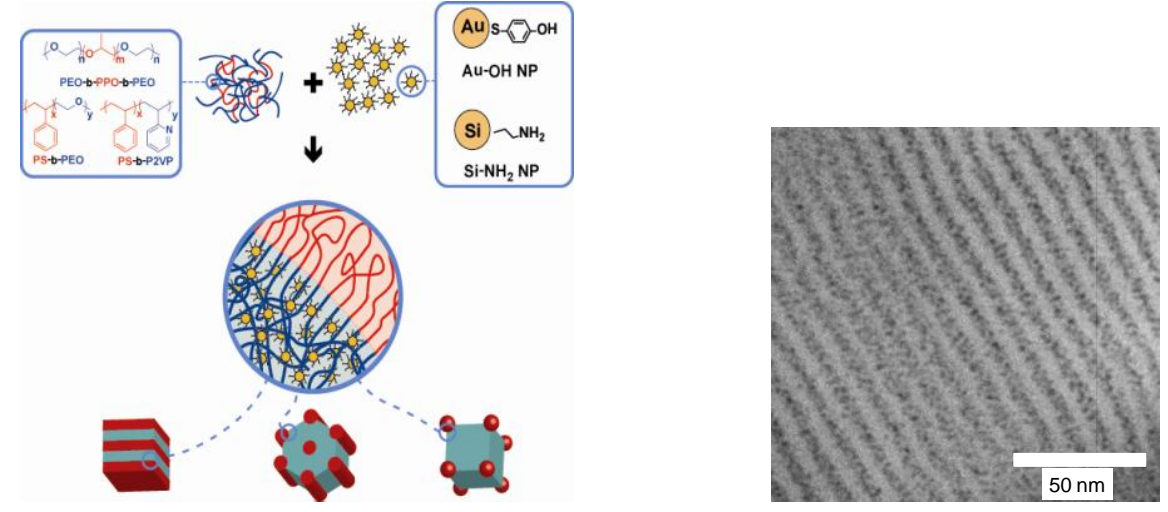

Figure 3: (Left) Scheme for additive-driven assembly of ordered hybrid materials, and (right) example of ordering of functionalized Au nanoparticles at high loadings.

\section{Nanoimprint Patterning}

Nanoimprint lithography (NIL) and patterning is a technique that has shown significant promise for high-throughput nanopatterning for a range of device applications. NIL's potential has garnered sufficient attention to place it as a future contender on the semiconductor industry manufacturing roadmap, and as a viable patterning tool for extremely high-rate production platforms such as roll-to-roll processing. Yet challenges remain for NIL in achieving both high-rate and large-area patterning due to the resist materials used for nanoimprint patterning. Widely used thermoplastic polymers have throughput limitations due to high viscosity even at elevated temperatures, resulting in 
the need for high pressures and long imprint times, along with the added cost of replacing damaged molds.

With goals of achieving patterned features of $50 \mathrm{~nm}$ at process rates of $5 \mathrm{~m} / \mathrm{min}$, Kenneth Carter of the CHM at the University of Massachusetts Amherst cited challenges that included both adhesion at the substrate level and long-term durability of ETFE molds in potential manufacturing processes involving UV-assisted roll-to-roll nanoimprint lithography (R2RNIL). Approaches to resolve these challenges include a focus on mold release and improving the etch resistivity of the resist materials. Development of new mold materials such as PFPE would enable R2R patterning of sub-100 nm features while further enabling the development of new processes for device fabrication such as multilayer coating, imprinting, reactive ion etch, metal deposition and lift-off on a R2R platform.

Jay Guo of the University of Michigan discussed R2RNIL efforts using UV curing of ETFE-patterned resists. He further described the development of custom photopolymer resins that were tailored to optimize substrate adhesion and minimize adhesion to the mold. Using glass molds, high-fidelity features having periods of $200 \mathrm{~nm}$ were demonstrated at unprecedented rates of $10 \mathrm{~cm} / \mathrm{sec}(6 \mathrm{~m} / \mathrm{min})$. Features with added edge roughness having periods as small as $100 \mathrm{~nm}$ were also demonstrated. The R2R NIL platform included in-line vacuum coating of metals to facilitate the fabrication of a range of value-added functional nanostructures and surfaces, including transparent conductor grids, plasmonic color filters, organic photovoltaic devices, and optical metamaterials. Another method of creating continuous nanopatterned lines such as grating structures was described. The technique, referred to as continuous dynamic nano-inscribing (DNI), utilized a rigid mold structure, such as grooves etched in a silicon substrate, as a mold pattern which is then heated and drawn across the moving web at speeds of up to 10 $\mathrm{cm} / \mathrm{sec}$. The grating patterns scribed in the photopolymer coating on the moving web are then UV-cured, resulting in a continuous, seamless grating pattern from beginning to end. While this approach is limited to straight-line features, the feature size and fidelity, as well as the rates achieved, are impressive.

Nikos Kehagias of the Catalan Institute of Nanotechnology (ICN) in Barcelona discussed efforts at ICN utilizing thermal NIL to fabricate a range of structures primarily for optical applications. He described ICN's participation in the European Union-funded NaPANIL program, consisting of seventeen partners from eight countries that are developing various aspects of NIL technologies for applications involving the control of light through surface properties, such as planar diffractive optical elements, light directional elements, emissive heads-up displays, and waveguide backlight sources. Typical features patterned using thermal NIL were limited to $100 \mathrm{~nm}$, with key challenges including the ability to maintain uniform features over large areas. The work at ICN as part of NaPANIL incorporated the entire value chain for R2R NIL, including application design, process modeling, creation of maser and working stamps, NIL processes on a moving web, and process monitoring. Incorporating such a value chain approach, process development and validation times for target applications were significantly accelerated for lab-scale (300 mm) R2RNIL manufacturing. 

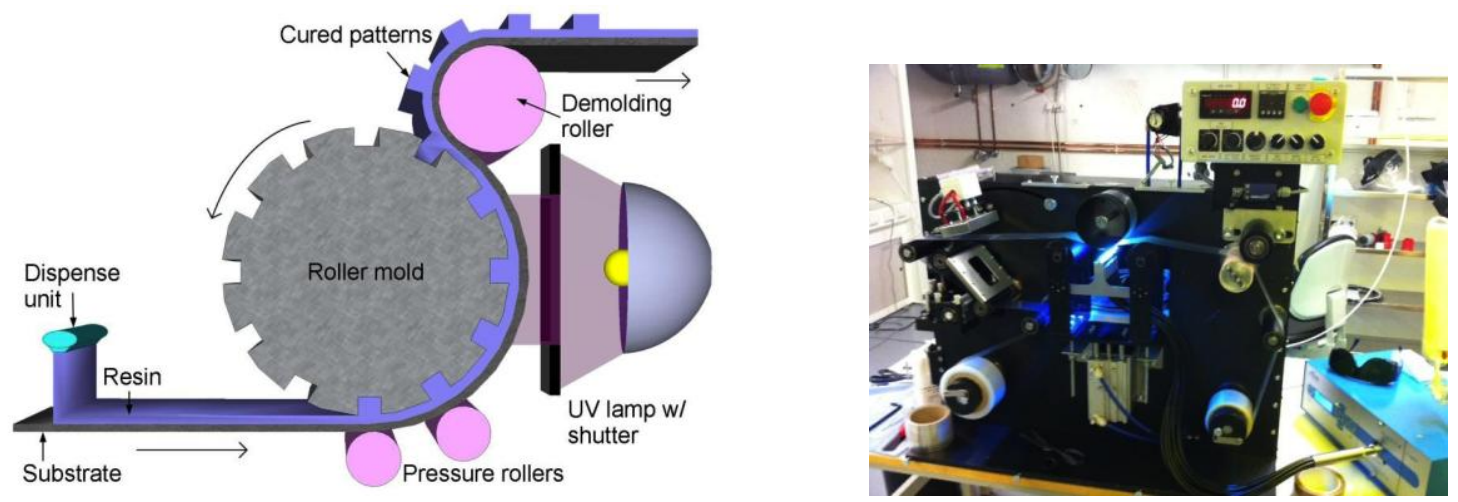

Figure 4: (left) Schematic of UV R2RNIL patterning method, and (right) image of R2RNIL tool at ICN-Barcelona.

Providing a similar perspective for R2RNIL research activities in Singapore, Hong Yee Low, of the Institute of Materials Research and Engineering (IMRE) under the Agency for Science, Technology and Research (A*STAR), described research focused on functional nanosurfaces. Utilizing direct surface patterning to form permanent nanostructures, IMRE research in this area is targeting a range of biomimetic surface functionalities, including superhydrophobicity, structural color and dry adhesive film, imparted through structural control rather than chemical composition. IMRE has recently launched focused projects in technical areas including R2RNIL, with further research and development in areas such as scaled manufacturing aspects of R2RNIL, including defect analysis and scaled device fabrication. The work on defect analysis for R2RNIL platforms has established a reference database of speckle-images to identify defect type, with capability for identifying the defect classification.

Dennis Slafer, another Polaroid alumnus now with MicroContinuum, Inc., discussed efforts incorporating R2RNIL in combination with additive/subtractive processes to fabricate integrated devices and functional structures. Utilizing in-line vacuum processes such as multi-target sputter coating and reactive ion etch, a range of value-added nanostructures could be synthesized for applications including optical data storage, hybrid transparent conductors, thin-film transistor electrodes, nano-antenna energy harvesting arrays, and metamaterials/metastructures for frequency-selective surfaces.

From a tool capability perspective, S. V. Sreenivasan from Molecular Imprints/University of Texas-Austin, described developments using inkjet-based R2R nanoimprinting. Based on the Jet \& Flash Imprint Lithography (J-FIL), a capability had been demonstrated that incorporated inkjet technology for efficient dispensing of materials combined with UV exposure to polymerize the imprinted features. This capability has demonstrated a superior lithographic patterning tool for a range of applications resulting in a reduction in both materials costs due to the efficiency provided by controlled dispensing via the inkjet head, and reduced capital costs in comparison to traditional lithographic tool requirements. Having demonstrated unprecedented performance for application in nonvolatile memory, semiconductor and bit-patterned media lithographic patterning, the JFIL approach was extended to an R2R platform. Example applications motivating this development included large-area amorphous silicon photovoltaic and electromagnetic field polarizers for displays, where patterned features in the 50-100 nm range provide significant performance enhancements. The resulting performance of the tool included 
sub-50nm resolution with a current resist aspect ratio of $\sim 3: 1$. Template longevity consisted of hundreds of imprints with a six-month goal of thousands of imprints. The JFIL R2R tool was well suited for high-quality pattern transfer and thin uniform RLT, having demonstrated $\sim 20 \mathrm{~nm}$ mean, $<5 \mathrm{~nm} 3 \mathrm{~s}$, with targets of $<15 \mathrm{~nm}$ mean. The platform demonstrated the potential for very high throughput exhibiting $\sim 1.5 \mathrm{sec}$ fluid filling and separation time, with a six-month goal of $<1 \mathrm{sec}$. The tool further demonstrated the ability to protect the film before and after nanopatterning. Several patterns were demonstrated with features approaching $50 \mathrm{~nm}$, with continuous replication over tens of meters and hundreds of imprints with no pattern degradation.

Many issues were mentioned by discussants as key barriers and challenges for R2RNIL to overcome for commercial scale-up of R2RNIL processes. These included limited availability of literature data, lack of proven industrial-scale R2RNIL tools, and challenges for large-area roller mold fabrication from the platform standpoint. These issues were cited as critical as there is a high interdependency among tool design, roller mold, process capability and materials in determining throughput and process design. Other issues discussed included suitability of resist materials (UV and thermal) and availability of web materials, including any form of materials standards. From the process tool and capability standpoint, several items were cited including large-area, costeffective e-beam patterning tools and capabilities, plasma etching tools for large-area, uniform R2R processing, ink jet applicators compatible with a wide range of UV monomers, the need to develop high-quality Ni electroforming processes for high aspect ratio, large pattern volume structures, the lack of high-durability, low-cost transparent imprinting molds or inexpensive/fast-replacement transparent molds, and the fabrication of seamless cylindrical imprint molds.

\section{R2R Process and Device Integration}

Process and device integration approaches will incorporate the value-added functionalities provided by engineered nanostructures and surfaces within a hierarchical device or system design. It is this focus that combines the expertise of $\mathrm{R} 2 \mathrm{R}$ processing with the batch processes developed for the semiconductor industry, and that capitalizes on scaling these processes to high-throughput and large-area. As mentioned above, several of the R2RNIL R\&D efforts included some form of vacuum coating, with the example of the MicroContinuum $\mathrm{R} 2 \mathrm{R}$ lines incorporating a full range of additive/subtractive processes to realize a range of devices and functionalities.

As an example of printed thin-film devices in a production environment, Jennifer Ernst of Thin Film Electronics ASA described the commercial development roadmap for fully-printed re-writeable memories based on ferroelectric thin film capacitor technology. Thin Film's roadmap includes the development of 20-bit re-writeable passive memory arrays, now being produced by an R2R manufacturing partner in South Korea, and extending the capacity to 128-bit arrays of both passive and addressable memory components. By extending the application areas to higher levels of integrated systems, Thin Film's product development approach will progressively lead to integration with other printed electronics systems, thereby unlocking new markets. As a pioneering venture manufacturing a printed memory technology, Thin Film is encountering numerous technical issues on the "frontline." Issues cited include surface roughness, barrier properties and deformation of substrates; compatibility and stability of materials 
sets and inks (whether UV-cured or sintered by thermal pulse exposure); and a lack of capability to achieve the required line features. This last issue was addressed using gravure offset and reverse gravure coating methods, but still had some limitations in producing the desired line features. In this example, the lack of machines to achieve fine patterns in both line height and width was cited, along with concern about the cost and capabilities of nanoimprint lithography. Other challenges cited for commercial development of printed memory products and applications included economics of materials development and scaling, access to a skilled workforce, and the necessary ecosystem to establish the value chain for negotiating product roadmaps. As such, it is necessary to find opportunities at the systems levels for integration of devices, and to further identify systems that use such devices. Resulting partnership models to foster commercialization would include a range of fundamental research, along with an application focus involving both small and large companies.
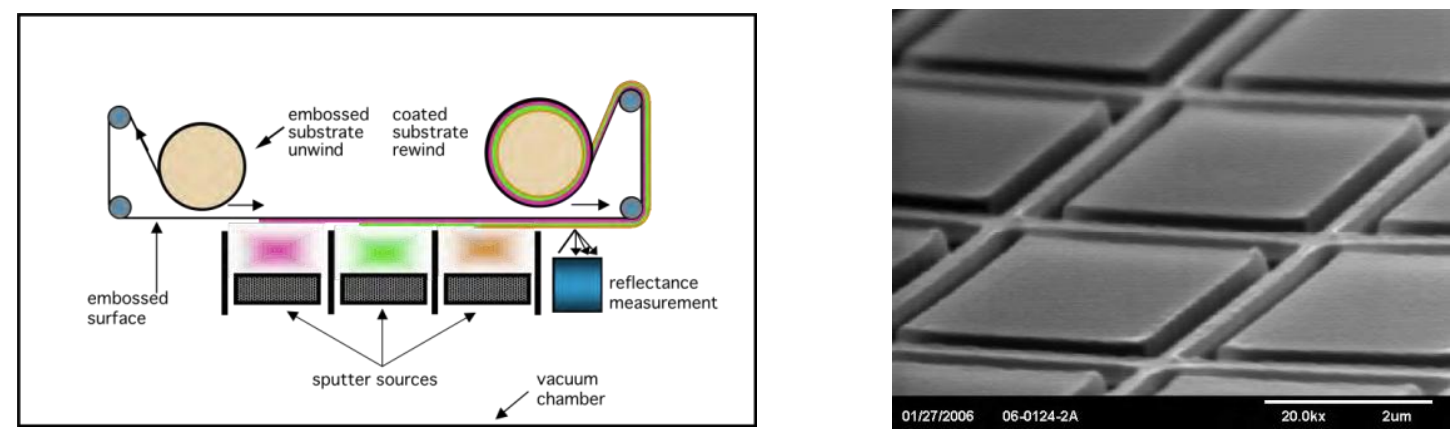

Figure 5: (Left) Schematic of inline vacuum coater, and (right) integrated device created using multiple nanofabrication steps.

Providing an overview of present facility capabilities to address manufacturing challenges of roll-to-roll fabrication of advanced electronic interconnects, Mark Poliks of Endicott Interconnect Technologies, and the Center for Advanced Microelectronics Manufacturing (CAMM) at Binghamton University, described CAMM's objective for providing R2R vacuum deposition, photolithography, and wet or dry processing of flexible, unsupported, thin film-based active and passive electronic devices and advanced interconnect technologies. Under this directive, CAMM participates with industry members and sponsors and academic partners to evaluate flexible R2R substrate materials and process capabilities for fabricating specialty prototype electronic substrates. The center will specify, design, develop and build future tool and processing equipment for the development of materials and processes for printed electronics. CAMM incorporates a range of advanced process tools within $\mathrm{R} 2 \mathrm{R}$ platforms, including vacuum deposition and plasma etch tools, inkjet coaters and a range of solution-based additive/subtractive processes. CAMM undertakes the continuous development of tools, processes and facilities in support of a range of device and process applications, with accomplishments in R2R photolithography and wet processing of 2-5 $\mu \mathrm{m}$ sized features on unsupported flexible plastic with 1-2 $\mu \mathrm{m}$ overlay and registration, R2R sputter and reactive sputter of $\mathrm{Al}, \mathrm{Cu}, \mathrm{Cr}, \mathrm{Ti}, \mathrm{Si}, \mathrm{Si} / \mathrm{Al}, \mathrm{Si} / \mathrm{B}, \mathrm{Zn}, \mathrm{InSn}$ and their oxides, for fabrication of top-side flexible sensors, medical electronics, capacitors, optical waveguides and other devices of interest to members and sponsors. CAMM also engages in fabrication of top-side multi-layer semiconductor coatings on unsupported flexible 
plastic films, and preliminary integration of TFT prototypes.

\section{Emerging Tools, Capability, and Infrastructure for R2R Platforms}

Nanomanufacturing processes for R2R platforms are further supported through a batch fabrication paradigm in terms of emerging process tools, design for manufacturing methodologies, and the necessary ecosystem and value chain that sustains the advancement and acceleration of this manufacturing technology platform. The workshop included discussions in each of these topical areas as described below.

\section{Emerging Processes and Tools for R2R}

\section{Atomic Layer Deposition}

Ganesh Sundaram of Cambridge NanoTech provided an overview of activities in the development of Atomic Layer Deposition (ALD) tools for R2R platforms. ALD is a thin film deposition technique in which films are grown by the sequential pulsing of chemical precursors onto the surface of a substrate. A typical process sequence involves introduction of precursor $\mathrm{A}$, followed by a system purge, then introduction of precursor $\mathrm{B}$, followed by another system purge, after which the steps are repeated. The precursor reactions on the substrate surface lead to the growth of the thin film on a layer-by-layer basis, with the resulting film thickness controlled by the number of cycles of the process sequence. As the deposition process is self-limiting, the films are extremely uniform, pinhole free, and exceptionally conformal. ALD is capable of depositing a range of metal oxide films, as well as a limited number of metal coatings, and has the further advantage of relatively low-temperature processes and reasonably low-cost precursors for most applications. As a result, ALD is finding traction in the semiconductor industry, and has further been scaled to large-area substrate processes for thin film photovoltaics and displays where the metal oxide coatings yield superior barrier coatings and dielectric films. As ALD processes are compatible with low-temperature organic electronics and substrates, a natural extension is to develop R2R platform capabilities. The key difference for adaptation of ALD to a moving web is the need for spatial separation of the process sequence described previously in contrast to the temporal separation. Thus, zoneseparated ALD is based on spatial separation of pulses rather than temporal separation of pulses. This alleviates the major time component of temporal ALD, namely the purge time, resulting in a process compatible with R2R schemes and providing a path to highly industrialized applications.

The spatially-separated ALD deposition cell is comprised of two main parts: a manifold supplying the precursor carrier and purge gases, and the deposition cell itself directing the gases to the substrate. Basic concepts for zone-separated ALD first explored an analytic model suggesting strong cell precursor isolation, in qualitative agreement with a more rigorous chemical fluid deposition model. Further use of RGA techniques as a diagnostic to measure cell crosstalk provided adequate characterization for design improvements, resulting in cell designs having minimal crosstalk $(<1 \%)$, achieved through mechanical enhancements and process optimization. Present physical system capability can coat at web speeds of $10 \mathrm{~m} / \mathrm{min}$. 


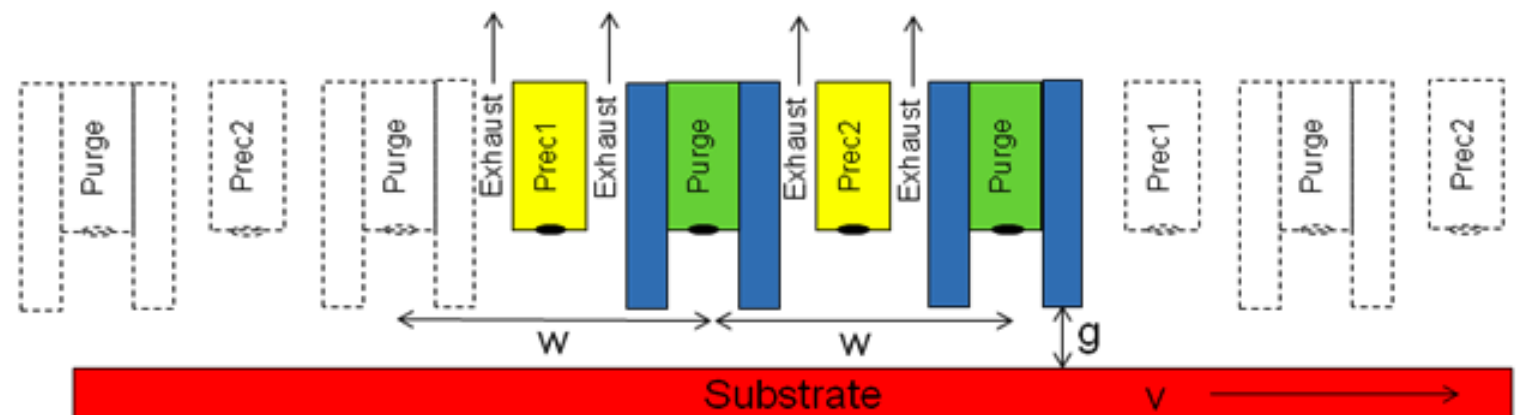

Figure 6: A simplified spatially-separated ALD unit cell configuration (one full ALD cycle) can be comprised of two reactants (precursors 1 and 2) separated by purge gases.

\section{Potentiometric Stripping Analysis for Electroplated Alloys}

Electroplating represents an additive, solution-based deposition process suitable for $\mathrm{R} 2 \mathrm{R}$ platforms for a range of metals and alloys. A key challenge for continuous, high-speed coating systems is the control of stoichiometry and the depletion the plating baths in webbased systems. Trevor Niblock of Magzor, Inc. described the development of R2R electroplating tools incorporating potentiometric stripping analysis (PSA) techniques to precisely control both stoichiometry and uniformity of metal and alloy coatings on flexible webs. Keys to maintaining sufficient process control in the electroplating steps included keeping the solution at the work surface fresh and evenly biased by agitating the bath, providing adequate circulation, further utilizing an inert environment such as an argon blanket to minimize the effects of oxidation, and utilizing a separate anode for precise control of field distribution. Utilizing the PSA approach, uniform coatings of various alloys have been demonstrated for complex, integrated structures. The tool further represents a rapid prototyping tool for flexible substrates, with initial applications demonstrated for magnetic actuators, and integrated systems for EMI shielding.

\section{High Temperature Roll-to-Roll Processes}

A primary aspect of $\mathrm{R} 2 \mathrm{R}$ fabrication discussed has been low-temperature processing compatibility. Even though processes and tools described thus far have been relatively low-temperature, there remains a need for substrate materials having higher $\mathrm{T}_{\mathrm{g}}\left(\sim 250^{\circ} \mathrm{C}\right)$ and lower thermal expansion characteristics. Even with these improvements, active devices for flexible electronics have limited performance resulting from the relatively poor electronic transport properties. Amit Goyal of Oak Ridge National Laboratory described the development of high-temperature $\mathrm{R} 2 \mathrm{R}$ processes suitable to create crystalline, high-performance semiconducting materials. The high-temperature process capability can exceed $1200^{\circ} \mathrm{C}$ through the use of a high-temperature metal or suitable substrate; calendaring of thin film coatings then occurs, followed by a thermal pressing step. Because typical processes tailor a series of functional layers, interdiffusion becomes a significant concern. This issue is resolved by depositing a stack of buffer layers to provide the required crystal orientation that include deposition of a diffusion barrier and then active layer coating. The high-temperature R2R processes described can be used to develop hybrid solution-based approaches as well. The high-temperature processes are suitable for a range of thin film crystalline materials, including silicon for solar PV, diamond, and other semiconductors. This R2R process capability opens up opportunities 
for large-area, high-quality semiconductors having electronic transport properties approaching those of bulk materials, thereby enabling high-performance electronic devices and systems.

\section{Metrology and Inspection}

Metrology remains a key potential limitation for R2R nanomanufacturing due to stringent requirements for monitoring nanoscale features at extremely high rates. Examples of target requirements include:

- Fast, convenient, non-destructive

- Resolution $<10 \mathrm{~nm}$

- Lateral dimension and height of the 3-D structure

- Suitable for in-line or in-situ operation

Nikos Kehagias of the Catalan Institute of Nanotechnology (ICN) in Barcelona discussed approaches being explored at ICN, including optical sub-wavelength diffraction with resolution of $\sim 50 \mathrm{~nm}+/-5 \mathrm{~nm}$ for both lateral dimension and height, and photoacoustic metrology having depth resolution/acoustic wavelength of $\sim 10 \mathrm{~nm}$, providing both dimensional and physical information for the structures being interrogated. Subwavelength diffraction utilizes test structures made of blazed gratings of sub-wavelength sized lines, enabling evaluation of the quality of imprinted sub-wavelength features in blazed grating (e.g., line-width, height, defects, sidewall angle, curvature) that affect the relative intensity of diffraction orders in far-field. The technique has the advantages that it is suitable for either transparent or opaque structures, is non-destructive, and allows rapid collection of data. Sub-wavelength diffraction analysis can be implemented by an optical microscope configuration for in-line metrology utilizing a collinear optical path for the incident and diffracted light, thereby allowing the entire diffraction pattern to be collected through microscope optics, reducing the space and time requirements for measurement. In this configuration, process diagnostics are suitable for use with alignment and imaging optics for in-line or in-situ measurements, as well as for defect detection.

Hong Yee Low of IMRE described the assessment of defects using post-pattern inspection from which a database was established of defect type and classification. Utilizing speckle-image analysis, the database identifies the specific defect types resulting from trapped gas, particles, pull-outs, and missing patterns for both mold and imprinted patterns. The defects were further characterized in terms of size, detection ability, probability of occurrence, and temporal definition. Based on this analysis, particles remained the highest probability defects.

Xian Du of the Massachusetts Institute of Technology discussed research in the David Hardt and Brian Anthony groups at MIT on metrology and inspection for process control for roll-to-roll and transparent substrates. The approach described investigates the use of super-resolution (SR) imaging in developing a tool to acquire optically-averaged (aliased) observations of nanoscale features in order to measure variations over large-area moving webs. Noting the need for inexpensive sensors suitable for large-range and highresolution--given that product miniaturization drives $\mathrm{R} 2 \mathrm{R}$ nanoimprint patterning and 
manufacturing--SR imaging techniques use a rapid scanner that can acquire multiple lowresolution (LR) frames over the same region of a work piece or substrate. Image mosaic techniques can then reconstruct the high-resolution, large-range SR image of the substrate by restoring the lost high-frequency features or patterns from LR images. By controlled variation of the conditions of the image acquisition state to capture a set of raw videos, the LR image data can be used to estimate a three-dimensional map of the observed film with a 10x increase in resolution. Using these variations, SR methods can reduce errors due to aliasing and under-sampling. Issues associated with adapting SR imaging to R2R moving webs include the translational motion that introduces vibration; this downgrades inspection speed and quality, and consequently translational scanning cannot generate a full-rank reconstruction matrix. By comparison, rotational scanning has the potential to reduce vibration, improving the acquisition of variations for high-frequency pattern reconstruction, and increasing the field of view. To address the non-uniform distribution of the scanning positions/information in polar coordinates, the design of an innovative rotational sampling method and inspection system for R2R manufacturing was developed using a set of constraints for rotational sampling which preserves the Cartesiancoordinates neighborhood. To demonstrate this, a rotational manufacturing inspection system was designed to integrate the rotational sampling algorithm on rotational stage. The rotational manufacturing inspection system integrated a controller, an open-loop rotational stage stacked on top of the $\mathrm{X}-\mathrm{Y}$ air bearing stages (allowing rotation of the part around a rotary Z-axis with a resolution of $0.0002^{\circ}$ ), and a high-speed area-scan camera with high-signification lens and high-speed frame grabber (500 fps). Testing for the SR sampling and control technique in the R2R manufacturing process used a three-roller test frame emulating a moving web. The test frame included a drive roller attached to a motor, an idler roller, and a hanging tension roller with independently controllable tension and position/velocity. Initial results demonstrated an innovative rotationsampling-based SR imaging platform that has the capability to achieve high frequency and wide field of view. Utilizing a fast-speed inspection system that incorporates rotational scanning to overcome the vibration in translation scanning facilitates the implementation of the rotational sampling in the $\mathrm{R} 2 \mathrm{R}$ manufacturing process. This technique further enables high-speed 3D profiling methods for transparent R2R products. Future plans include building an R2R manufacturing prototype to test these metrology and process modeling methods to be implemented on the metrology frame for continuous interrogation of a moving web on the R2RNIL tool at the University of Massachusetts Amherst.

Ken Carter of the CHM at UMass Amherst further described efforts to incorporate metrology on the test frame platform there. In addition to collaboration with MIT described above, UMass is collaborating with Alex Liddle and Gregg M. Gallatin of NIST to develop a device for measuring long- and short-range order in block copolymer films. NIST's approach was to investigate the use of flying height sensor arms, similar to hard disk drive read heads, that float a few nanometers above the moving web. Laser gauges are employed to track the relative position of each sensor arm with nanometer precision. The sensor arms automatically (aerodynamically) move up and down to track vertical deviations in the top surface position of the web, thereby measuring the specific type of pattern(s) being generated on the web. The UMass/CHM test frame provides a platform to validate these emerging metrology and inspection tools in a robust, 
industrially meaningful and scalable environment.

\section{Process Modeling and Control}

Joe Petrzelka of the David Hardt group at MIT discussed an investigation of scale-up of soft lithography to R2R technology. With a focus on modeling and control of the contact region for R2RNIL platforms, the investigation was represented as a multiscale problem equating force, pressure, and contact area for local deformation of features, and macroscale deformation of the imprint master in order to better understand the critical points for pattern collapse. R2RNIL process models allow a better understanding of frequency and source of errors in the imprint process. Examples of error sources included cylindricity, bending under load, and thermal distortions imparted on the roll; mold errors, thermal shrinkage and ink swelling in the stamp; and planarity, surface roughness, bending, and thermal distortion in the substrate. Error frequency ranges from low to high and is associated with sources such as alignment, stamp-body-to-roll distance, feature scale discontinuities, and nanoscale surface roughness, respectively. In order to control the contact an effective sensing and control feedback loop is necessary. The sensing is accomplished by coupling a quartz prism to the soft mold through which infrared imaging is conducted. Control is accomplished by using images enabling both spatial filtering feedback compensation for the contact controller, and state feedback compensation for position control. For scaling to production level processes, error mapping for feed-forward process control would utilize the transparent substrate method to map the errors in any particular stamp, given an upstream measurement of substrate error. In-process control would utilize a downstream inspection to monitor temporal drift of stamp.

R2RNIL represents an enabling process with growth opportunities in metastructures and high-resolution device patterning. While numerous real mechanical engineering challenges exist, none are show stoppers. In-line process sensing and control are critical capabilities for utilizing robust patterning. Near-term challenges for process control include mid-range error rejection, stamp production and mounting techniques, and fullscale platform integration incorporating inking, steering, and etching processes.

\section{Manufacturing Scale-up and Commercialization}

The workshop presentations by industry, government, and academic participants consisted mostly of research and development activities in scaling materials and device technologies to laboratory-scale R2R platforms utilizing approaches that are relevant for further scale-up to commercial production platforms. While examples of commercial production incorporating nanofabrication techniques were presented, and further exist elsewhere both in the U.S. and globally, the level of complexity and integration remains limited both in functionality and value-added. To accelerate growth of materials, process and platform technologies, a robust and sustainable ecosystem must be established. The basis for such an ecosystem will translate from existing infrastructure in the R2R print and coating industry, adopting emerging nanomanufacturing methods, materials and tools to create future value-added consumer products having unprecedented functionalities. Aspects of such an infrastructure to accelerate an advanced manufacturing agenda in $R 2 R$ nanomanufacturing were also addressed during the workshop. 


\section{Standards}

Daniel Gamota pf Printovate Technologies presented an overview of the IPC Printed Electronics Standards development initiative. Standards are an important aspect of the successful commercialization of any product, and typically are underestimated. Benefits of standards include building end user confidence, creation of a common language between producers and users, promotion of product compatibility and interoperability, overcoming trade barriers to open markets, and fostering diffusion and adoption of technology. As printed electronics and R2R nanomanufacturing transcend from fundamental science to laboratory-scale production platforms within academia and industry, the transition to pilot-scale production is hampered by several factors that can benefit from standards. The resulting delay in the commercialization cycle includes losses resulting from incoming materials variations, process technologies, process tolerances, equipment and operator inconsistencies, and lot-to-lot variations, making it extremely costly and difficult to scale to R2R production capacity. Standards are necessary to assist in the translation of discrete processes to an integrated manufacturing flow. Applying design for manufacturing principles to deliver robust processes and high final product yield will minimize loss in performance, and accelerate the commercialization cycle. Key standards areas relevant to R2R nanomanufacturing include terminology, test and measurement standards (for materials, processes and manufacturing), structures (conductors, resistors, capacitors), inks (copper, silver, aluminum, CNTs), substrates (paper, plastic, metal), encapsulation/barriers, printing processes, and structures for conductive traces/wires. Applications areas include diodes, memory, backplanes, and RFID.

In developing standards, a committee will typically leverage existing standards to help craft new standards. An example was given of a standards development subcommittee for printed electronics materials. The goal is to develop procurement documentation and standard incoming inspection procedures of base (substrate) material for issues such as cleanliness, roughness, surface energy, ink spreading on flex surfaces, hydrophilic/hydrophobic properties, dot gain, and edge definition. In order to establish this, the subcommittee must identify critical substrate properties and generate specification sheets for different substrates. If possible, it must further develop standard test vehicles and methods. Similar standards activities must address all of the topics specified above relevant to $\mathrm{R} 2 \mathrm{R}$ nanomanufacturing, thereby providing a consistent base infrastructure for subsequent product development and scaled manufacturing.

\section{Accelerating Technology Transfer and Advanced Manufacturing Scale-up}

Core to the innovation cycle in the U.S. is technology transfer from laboratories conducting fundamental and applied research to industry seeking to commercialize advanced products for consumer markets. Technology transfer inevitably requires product/process proof-of-concept, design optimization, pilot production, and scaled manufacturing leading to commercialization of the final product. The National Nanotechnology Initiative (NNI), supported by numerous reports by the scientific advisory community, has stressed the need for an enhanced focus on effective technology transfer, nanomanufacturing and commercialization as a means to create a return on investment for the U.S. government's support of nanotechnology research and development over the past decade. An important aspect of this is the generally accepted 
theme that the U.S. economy relies heavily on small businesses and that much development of nanotechnology is occurring in small businesses. In order to foster successful growth of small and medium enterprises (SME) engaged in nanotechnologybased products, new paradigms must be established that enable technology research and development to occur and for the SME to overcome the so-called "valley of death" scenario. This scenario, in which the proof-of-concept or feasibility has been demonstrated, yet the path to commercialization, scaled manufacturing and sustainability remains in the distance, is potentially prohibitive to a robust, nanomanufacturing ecosystem, mainly due to the long time to market for many nanotechnology products. In this respect, federal and state funding for small companies has a significant impact, since venture capital funding is typically unavailable to early-stage hardware technology companies, and seed/angel gap funding may be too expensive. In discussing this gap for accelerating commercialization of R2R nano-enabled technologies, James Watkins of the University of Massachusetts Amherst described the recently launched $R 2 R$ nanofabrication facility at the Center for Hierarchical Manufacturing. Supported by an Industrial Advisory Board from the R2R sector, a cluster organization has been formed to address specific technology issues of concern to cluster members. The facility and industry cluster further provides industry training and access to the equipment.

Another concern that may have a long-term impact on technology transfer and collaboration from academic and government research institutions involves the intellectual property maze that exists, given the number of players and technologies. While these issues have not impacted the innovation cycle or development of intellectual property as evidenced by significant increases in nanotechnology-related patent filings and scientific journal publications, they may grow in significance as more nanotechnology-enabled products enter the marketplace.

\section{Pilot Line Facility/Manufacturing Hub}

The NNI has described a strategic mission to make available facilities with sophisticated capabilities to support nanotechnology innovation in the U.S. The National Nanotechnology Infrastructure Network (NNIN), consisting of thirteen user facilities located at universities within the U.S., provides fabrication capabilities and equipment that users can access for a sliding fee based on company size. Unfortunately in today's environment, many small companies don't have the resources to take adequate advantage of such facilities. Furthermore, while the facilities provide fabrication processes and sophisticated tools and equipment to conduct proof-of-concept work, NNIN facilities often lack emerging nanofabrication processes, as well as scaled platforms leading to pilot scale manufacturing. As a result, this typically leads to slower development cycles and a distraction for the technologist trying to deliver "nanotech" in a form that can move into manufacturing. The NNIN facilities are complemented by user facilities at numerous FFRDCs (DOE, NIST, DoD), which have somewhat different models for industry participation, and in many cases provide more value-added from the standpoint of facility capability and resources. In either case, for effective technology transfer that leads to manufacturing scale-up some investment by the industry partner is necessary.

In considering potential partnerships for accelerating progress toward volume manufacturing, academic-FFRDC-private pilot line facilities organized through a consortium or "technology hub" model have the benefit of enabling industry to work 
closely with academic and government researchers to evaluate and transfer newlydeveloped lab concepts to meaningful pilot and manufacturing environments. Pilot line facilities enable the development, demonstration and optimization of full processes, and remain a vital step between lab coupon demonstration and production line. The ability for industry to understand and validate emerging nanomanufacturing technologies in this type of partnership diffuses critical knowledge in both directions, while reducing risk and cost. A consortium-based organization further enables SME participants to access a wide range of partner capabilities and expertise to achieve their commercialization goals, Increased federal support for small company participation can facilitate such partnerships, but an effective business model that protects intellectual property while enhancing the broader knowledge sharing between all participants is also necessary.

\section{Associated Consortia Models (EU and Asia)}

Hong Yee Low of IMRE described the Industrial Consortium on Nanomprint (ICON), involving industry participants ranging from SMEs to big companies, with an objective to foster the sharing and transfer of nanoimprint technology with industries, while further enhancing commercial readiness and adoption of the technology through synergistic partnerships. The consortium model takes a holistic approach to address problem-solving, delivering to consortium members a detailed evaluation of roll-to-roll nanoimprinting that can be carried over into a manufacturing context. IMRE has recently launched focused projects in technical areas including R2RNIL. The IMRE projects have further conducted defect analysis for R2RNIL platforms, establishing a reference database of speckleimages to identify defect type and classification. Some of the benefits provided by ICON participation are global perspectives through transnational collaborations; sharing of know-how accumulated; availability of tools, materials, and processes to participants; and future availability of simulation and monitoring tools.

Nikos Kehagias of the Catalan Institute of Nanotechnology (ICN) in Barcelona described ICN's participation in the NaPANIL program funded by the EU, which consists of 17 partner Institutions from 8 countries developing various aspects of NIL technologies for applications involving the control of light through surface properties such as planar diffractive optical elements, light directional elements, emissive heads up displays, and waveguide backlight sources. Each partner institution had numerous industry partnerships in different applications areas, and provided a mostly open access to process information and results. NaPANIL has posted a NIL process database on its website (www.NAPANIL.org) to better diffuse the knowledge gained from the public-private partnership. Several of the participating institutions have ramped up efforts in pilot-scale process development to assist industry with the next steps in commercialization and scaled process research and development.

\section{Summary of Key Challenges}

Several key challenges exist for adopting R2R nanofabrication methods and practices to manufacturing platforms in the U.S. and globally. While flexible electronics products are beginning to incorporate nano-enabled components within device or system architectures, the sophistication and level of integration remains limited. A prime example is transparent conductors for touch screens made from solution-cast silver nanowires or carbon nanotubes. As a nano-enabled intermediate, the cost and performance of the 
transparent conductors is sufficient for present market competition, and is beginning to replace ITO in select products, with an assurance of gaining further market share as highvolume $\mathrm{R} 2 \mathrm{R}$ processes and associated reliability practices and manufacturing materials and process standards are adopted. Yet this is just one example of a nano-enabled solution-cast product navigating the road to achieving mass-market adoption, and that still remains in the distance. To accelerate the transformation of nano-enabled fabrication methods and materials, the key challenges must be identified and addressed. These key challenges include materials and process costs, planarization and base/barrier layers, including transparent conducting films, coatable dielectrics, the creation of ordered nanoscale hybrid materials as active layers via directed- and/or additive-driven selfassembly, and continuous device-level patterning using roll-to-roll nanoimprint lithography.

R2RNIL barriers and challenges include the limited availability of literature data, lack of proven industrial scale R2RNIL tools, and large-area roller mold fabrication from the platform standpoint. Additional barriers include suitability of resist materials (UV and thermal) and availability of web materials, including any form of materials standards. From the R2RNIL tool and capability standpoint, barriers include the availability of large-area, cost-effective e-beam patterning tools/capabilities, plasma etching tools for large-area, uniform $\mathrm{R} 2 \mathrm{R}$ processing, and ink jet applicators compatible with a wide range of UV monomers. Challenges include the need to develop high-quality Ni electroforming processes for high aspect ratio, large-pattern volume structures, and the lack of highdurability, low-cost transparent imprinting molds or inexpensive/fast replacement transparent molds, along with the fabrication of seamless cylindrical imprint molds.

Further challenges relating to patterning and process integration include identifying product specifications, the high expense of materials, understanding product performance tolerance to patterning-related defects, the need for clean manufacturing, rate control and purity levels for nano-morphologies, ensuring that materials of interest are compatible with flexible supports and the required patterning method, the use of primer layers and treatments that enhance resolution and/or adhesion, thermal and environmental stability of continuous, flexible substrates when processing functional layers, registration of multiple layers on flexible substrates, dimensional stability of flexible supports, and barrier layers that minimize poisoning of functional layers or migration into flexible supports.

From the perspective of fostering innovation, enhancing U.S. competitiveness, and accelerating the commercialization of emerging flexible electronics products, key barriers include the availability of collaborative demonstration facilities and well-defined proofof-concept projects that would not only validate the R2R tool platforms, but facilitate the adoption of emerging processes and materials enabled through select device fabrication and integration examples (e.g., photovoltaics, batteries, flexible memory).

\section{Conclusions and Recommendations}

The workshop elucidated the market opportunity for flexible electronics, along with potential paths for enhancing U.S. competitiveness in these markets through incorporation of R2R nanomanufacturing methods and infrastructure. While the benefits of advanced manufacturing utilizing emerging process and materials platforms have 
significant potential, the present economic conditions and positioning of industry to gain market share for specific application areas remains a significant barrier to widespread development and adoption of these technologies. At the heart of these issues is the cost and risk involved to incorporate new processes that are not supported by a broader infrastructure involving standards, tools, materials, and reliability/process control through which next generation flexible electronics can be scaled for commercial manufacturing. As a result, adoption of emerging manufacturing processes is slow, leading small businesses and entrepreneurial innovators to delay technology progress, and diminishing the innovation cycle and business growth. Methods to enhance both the transition of emerging research results to industrially meaningful process platforms, along with manufacturing scale-up development of R2R nanomanufacturing processes, are necessary to bridge this critical gap between innovation and commercialization.

Recommendations for follow-up activities that would enhance U.S. competitiveness in R2R nanomanufacturing, thereby impacting the broader flexible electronics application areas, include a cohesive effort combining the need for standards, improved metrology and in-line characterization, scaled process control and modeling, materials, off-line characterization, and advanced tools. Further establishment of infrastructure, including possible shared demonstration facilities where academic research could be transferred and scaled to industrially meaningful process scales in close collaboration with industry, would accelerate the adoption of advanced nanomanufacturing processes. Establishing a relevant industry/academic consortium or innovation hub could both enable broad-based industry growth impacting numerous product and application areas as well as advance the performance of key intermediate technologies (e.g; barrier films, transparent conductors, high-k dielectrics, etc.) that impact a broad range of different product applications. Creation of an industry network to further elucidate and resolve such critical issues would be advantageous to U.S. competitiveness in this area. In this context, R2R nanomanufacturing would be an appropriate theme for an advanced manufacturing institute or center of excellence, either on its own or as part of a larger manufacturing focus.

\section{Appendix A: Participant Response to Workshop Questionnaire}

Several key questions were provided to participants in preparation for the workshop. These questions and subsequent responses are presented below.

Response to Key Questions

What are the biggest barriers or process bottlenecks that need to be addressed to realize roll-to-roll $(\mathbf{R} 2 \mathrm{R})$ nanomanufacturing?

$\mathrm{R} 2 \mathrm{R}$ manufacturing traditionally consists of coating and printing processes. While there are many companies engaged in $\mathrm{R} 2 \mathrm{R}$ manufacturing, there remains a general lack of standardized infrastructure in some cases, and most academic institutions do not have R2R fabrication facilities. As a result, for nanofabrication technologies, $\mathrm{R} \& \mathrm{D}$ data are still lacking on what are achievable with $\mathrm{R} 2 \mathrm{R}$ processes and what are the limitations, especially in the context of throughput. In combination with this is a lack of validated, multi-scale process models to compliment the fabrication facilities. Future programs, for example at universities or shared user facilities, should include integrative studies of machine mechanics, materials, and processes. With regard to 
emerging process technologies, such as nanoimprinting for example, there is a need to provide training to companies.

In addition to providing a connection between emerging $\mathrm{R} 2 \mathrm{R}$ process $\mathrm{R} \& \mathrm{D}$ and scaled manufacturing, several challenges related to process tools and core capabilities remain. These include:

- Large-area, cost-effective e-beam patterning tools/capabilities

- Plasma etching tools for large-area, uniform R2R processing

- Ink jet applicators compatible with wide range of UV monomers

- Development of high-quality nickel metal electroforming processes for high aspect ratio, large pattern volume structures

- High-durability, low-cost transparent imprinting of molds, or, inexpensive/fast replacement transparent molds

- Fabrication of seamless cylindrical imprint molds

- Large-area, real-time metrology and process characterization

Can you foresee enabling/emerging technologies that need to be developed in order to realize roll-to-roll nanomanufacturing?

The challenges in process tools and core capabilities cited above in the previous question represent enabling technologies impacting the extension of $\mathrm{R} 2 \mathrm{R}$ processes for a range of applications. For emerging technologies such as nanoimprinting for example, large-scale R2R tools have not been proven. Additionally, the supply chain has not been established. As a result, well-established cost and performance models are not available. In order for R2R nanomanufacturing to gain a foothold as a viable competitive platform for commercialization of value-added products, development in these key areas is critical.

In addition, further development of emerging process tools towards large area processes will be essential for realizing R2R nanofabrication.

- Precision ink jet fluid applicators

- Any dot, anywhere; high uniformity and thickness control

- Atmospheric plasma etching

- Lower-cost surface processing with elimination of vacuum step

- R2R-ALD (atomic layer deposition)

- Precision application of very thin layers at high rates

- High conformality; uniform coating of aspect ratios up to 1000:1

- High film density, low film stress

- Continuous, pinhole-free ultra-thin films

Are there specific materials and/or metrology needs that are needed to realize rollto-roll nanomanufacturing?

Materials developments are critical for the extension of $\mathrm{R} 2 \mathrm{R}$ nanofabrication 
processes to large area and high throughputs. In nanoimprint technology, the imprint materials need to be developed (especially for UV roll-to-roll imprint). There is also a limited supply of suitable web materials. Examples for a "materials wishlist" include:

- UV polymers that resist plasma crosslinking

- Transparent conductive polymers having:

- Higher conductivity and light transmission

- Improved durability/stability

- UV curable

- Less costly, higher-temp substrates

- $\quad(>250 \mathrm{C}$; preferably clear...)

In-line metrology is a must but it is still not clear what technique is going to work best. As such, metrology and inspection incorporating in-line optical techniques is presently being developed, but significant challenges remain for monitoring of high throughput processes having nanoscale features. In combination with this, modelbased real-time diagnostics and control would compliment the development of process modeling and control methods.

What infrastructure needs to be developed to transition roll-to-roll technologies to volume manufacturing?

In order to extend $\mathrm{R} 2 \mathrm{R}$ nanofabrication technologies to volume manufacturing, several infrastructural needs must be established relevant to emerging processes and tools. For example, parameters affecting throughput and defects control for various processes need to be established. In addition, the necessary supply chain is lacking, and needs to be broadly established, along with standards. Standards developments cannot be underestimated, and a more concerted effort in this area is necessary. One way to address these issues may be through the establishment of pilot line facilities for development, demonstration and optimization of full processes. This infrastructure component would provide a vital step between lab coupon-scale development and production line scale-up, ultimately reducing risk and cost, and providing a more rapid development path for product commercialization. This is due to the high cost of roll-to-roll instruments. This can be alleviated by creating shared facilities that will be accessible for academics and for industrial participants to try out some ideas, as well as establish emerging processes and materials for broader use. In this manner, the process development cycle can address key enabling technologies that are far upstream from large attractive markets, for example, large-format templates for R2R nanoimprint.

What sorts of partnerships (public, private, academic, consortia) are needed to accelerate progress towards volume manufacturing?

For an R2R nanomanufacturing program, industrial participation is more important than typical manufacturing programs in terms of transitioning emerging $R \& D$ onto large-scale production platforms. The question is what is the best model for this to happen such that both academics and industry can benefit from the program. One concept cited is the Fraunhofer Institute in Germany, which provides a fertile ground for industry to actively participate in manufacturing platform development, provide 
direct and indirect support, and directly benefit from their investment. To impact the R2R manufacturing base in the U.S., industrial partners must actively participate in the process development. Academic-FFRDC-private interactions and partnerships can evaluate and transfer newly developed laboratory concepts to pilot/manufacturing platforms. To better facilitate this, a R2R nanofabrication network, similar to the NNIN concept, could provide a multi-tiered benefit to the broader R2R manufacturing industry. In this concept, the focus should go beyond exploratory process and device studies, and should include studies of process reliability, robustness, and device integration and reliability studies. Shared facilities could be established by region, consortia, or possibly on technology/application lines. Shared facilities or consortia can access wide range of partner capabilities and expertise in order to address key challenges and share common goals. One component to better facilitate these consortia and expedite the transition of emerging nanofabrication processes would be increased federal funding for small companies. In conjunction with this, roadmaps should be established for significant end-applications. Roadmaps should be application-specific, as each application will have its own performance and cost targets, and ultimately the roadmaps could be aligned to the user facility focus and structure.

Additional themes impacting the transition to volume manufacturing for which public-private partnerships could better facilitate include workforce training and education, nano-EHS and risk assessment. These aspects are of critical importance to the industry community, and could be facilitated through such consortia or user facility networks.

\section{Appendix B: About the National Nanomanufacturing Network}

The goal of the NNN is to build a network of experts and organizations that facilitate and expedite the transition of nanotechnologies from core research and breakthroughs in the laboratory to production manufacturing. Partners and affiliates will find value added through a range of services including training and education, industrial vision and roadmap development, thematic conferences and workshops, and a comprehensive information clearinghouse.

In order to advance nanomanufacturing and competitiveness in the U.S., collaborations must be established between key resources and organizations to address challenges of significant national importance. While initial advances in nanomanufacturing will likely be an evolution of existing manufacturing practices and infrastructure incorporating nanomaterials and components, the long term vision will involve revolutionary processes and practices, tools, materials, and embedded metrology and analytical techniques. In order to achieve this long term vision, critical resources, capabilities, and knowledge must be brought together to advance the state-or-the-art.

To facilitate the necessary breakthroughs that will have high impact in the nanomanufacturing community, information exchange, resource partnering, and open discussion of ideas, discoveries, and best practices is necessary. Key challenges exist in providing an open platform for networking while protecting the proprietary information and intellectual property of the community. 
To accomodate the networking necessary to establish these collaborations, the NNN is presently building an alliance of members and affiliates that will facilitate the discussions, interactions, and critical teaming arrangements for development of nanomanufacturing testbeds and demonstrations. Examples of the NNN's role in fostering these collaborations include:

Understanding the nanomanufacturing needs of small businesses, large businesses, and industrial sectors.

Identify members having specific needs and connect them with members having potential solutions.

Establish a virtual user network with the NNN affiliates and develop appropriate guides for working with the user network.

Establish research testbeds and demonstrators for new nanomanufacturing techniques through the NNN affiliates.

Establish nanomanufacturing research roadmaps for specific industrial sectors.

Identify emerging nanomanufacturing technologies and design focused test platforms.

Provide an information exchange forum through the Internano website to enable direct communications between member organizations and individuals in the field.

The NNN encourages and promotes registration of individual and organizational resource information on it's directory in order to facilitate networking, sharing of timely information, and partnering of key resources. This self-maintained directory enables registrants to provide individual or organizational information on capabilities, needs, links, publications, patents, and processes.

In order to further enhance the value of the $\mathrm{NNN}$, the members and stakeholders in specific industrial and technology sectors are encouraged to provide this important feedback, either in the way of open forums on the Internano website, or through direct, individual contact to the NNN which will not be placed in open forums without the permission of the member. This key information helps the NNN to understand the short term and long term needs of the community and industrial sectors, and allows us to incorporate these needs into long term development and roadmap strategies.

The NNN will further provide academic outreach in the form of thematic conferences and workshops on a range of topics in the nanomanufacturing area. This outreach will further facilitate critical roadmap development between key industrial sectors and academic/government organizations that are stakeholders in the topical area, thereby providing the directions and first steps to establishing the nanomanufacturing capability for the community as a whole. These conferences and workshops will include experts in the field, and stakeholders in the topics being discussed, and will provide a high standard in quality for the topical presentations.

\section{Appendix C: About NIST}

Founded in 1901 and now part of the U.S. Department of Commerce, NIST is one of the 
nation's oldest physical science laboratories. Congress established the agency to remove a major handicap to U.S. industrial competitiveness at the time - a second-rate measurement infrastructure that lagged behind the capabilities of England, Germany, and other economic rivals. Today, NIST measurements support the smallest of technologies - nanoscale devices so tiny that tens of thousands can fit on the end of a single human hair - to the largest and most complex of human-made creations, from earthquake-resistant skyscrapers to wide-body jetliners to global communication networks. We invite you to explore our web site to learn about our current projects, to find out how you can work with us, or to make use of our products and services.

This section of our web site contains basic information about NIST. If you can't find the information you need, contact Public Inquiries at (301) 975-NIST or inquiries@ nist.gov.

General information -- links to program information, speeches, testimony, frequently asked questions.

Budget, planning, and economic analysis -- detailed budget information, strategic plan, economic analysis of NIST programs

Organizational chart -- shows NIST's organizational structure

Staff directory -- use to get the phone number, mailing address, or email address of NIST employees

A-Z subject index -- alphabetical listing of research topics, activities, programs, products, and services

No Fear Act policy

Small business contact

Visitor information

NIST Overview (video)

NIST Director's Office

About the Department of Commerce

Strategic plan and annual performance plan

Commerce and the Small Business Paperwork Reduction Act 